\title{
Aplicación de un ciclo de mejora docente en el aula en la asignatura de Biología (Grado de Medicina)
}

\section{Application of a cycle of teaching improvement in the classroom in the subject of Biology (Degree in Medicine)}

EnCARnación LOZANo PÉrez

Universidad de Sevilla

Departamento de Citología e

Histología Normal y Patológica

elperez@us.es

ORCID: https://orcid.org/0000-0003-0254-0935

DOI: http://dx.doi.org/10.12795/9788447231003.042

Pp.: 885-902 


\section{Introducción}

En esta comunicación se presentan los resultados de la puesta en práctica de un ciclo de mejora en el aula en el bloque de citoesqueleto celular, en la asignatura de Biología, obligatoria en el primer curso del grado en Medicina. Los objetivos de este bloque de contenidos teóricos es que los alumnos comprendan los elementos que forman parte del citoesqueleto, sus funciones y las interrelaciones que estos elementos entre sí. Precisamente estas interconexiones conforman una estructura altamente dinámica, que sirve a la vez como esqueleto estructural y como soporte al movimiento celular, tanto intra- como extracelular. Los resultados de este ciclo de mejora ponen de manifiesto que los estudiantes asimilan fácilmente contenidos de diversa índole cuando son ellos los que activamente toman parte en el proceso de aprendizaje.

\section{Breve descripción del contexto}

El ciclo de mejora se implementa en un grupo de estudiantes de 1er curso del grado de Medicina, en la asignatura de biología, obligatoria. El grupo está compuesto por 88 alumnos matriculados, de ellos alrededor de 20 repetidores, otros matriculados por primera vez tras completar la etapa del Bachillerato y algunos (pocos) procedentes de cursos de Formación Profesional. De todos los matriculados, asisten a las clases de forma regular alrededor de 60 alumnos. En general, son estudiantes muy motivados y vocacionales, que han hecho un gran esfuerzo académico para poder matricularse en el grado de Medicina. Debido a la situación sanitaria que atraviesa el país debido a la pandemia vírica, toda la actividad durante este ciclo se lleva a cabo online, a través de la plataforma Blackboard Collaborate proporcionada por la Universidad de Sevilla.

Ciclos de Mejora en el Aula (2020). Experiencias de Innovación Docente de la US Esta obra se distribuye con la licencia Creative Commons 


\section{Diseño previo del ciclo de mejora en el aula (CIMA)}

En este apartado se describe el diseño del CIMA, tal y como fue concebido antes de su puesta en práctica. La aplicación del CIMA coincide temporalmente con un bloque de temas relacionados con el citoesqueleto celular, en la asignatura de Biología, con un alto contenido conceptual y a priori con escasos contenidos procedimentales. Debido a ello, el ciclo de mejora abre la puerta para realizar dos mejoras fundamentales en mi enseñanza: primero, a priorizar contenidos fundamentales frente a los accesorios (ver mapa de contenidos), tal y como sugieren García-Díaz y cols., en su libro “Enseñanza Universitaria" (García-Diaz, Porlan y Navarro, 2017); segundo, a buscar metodologías que acerquen la asignatura a la vida cotidiana de los alumnos (secuencias de actividades) (Finkel, 2008).

\section{Mapa de contenidos}

El CIMA se centra en el citoesqueleto celular, compuesto principalmente de tres elementos: microtúbulos, filamentos intermedios y microfilamentos. Este hecho permite dividir en tres bloques el CIMA. Cada uno de los tres bloques comparte con el resto la misma dinámica de pregunta inicial, subpreguntas, actividades de contraste, actividad de síntesis, etc, como veremos a continuación.

El mapa de contenidos parte con una pregunta inicial, muy general, que engloba el sentido del CIMA en su conjunto: ¿es el citoesqueleto un mero soporte celular o su función es más compleja que esa? Esta pregunta pretende indagar sobre los modelos mentales que tienen los estudiantes adquiridos previamente sobre el citoesqueleto,

Ciclos de Mejora en el Aula (2020). Experiencias de Innovación Docente de la US Esta obra se distribuye con la licencia Creative Commons 
seguramente incompletos, y hacerles reflexionar sobre este tema. Intentamos contestar a esta pregunta desmenuzándola en pequeñas cuestiones pormenorizadas ya dentro de cada bloque de microtúbulos, filamentos intermedios y microfilamentos. El fin de las subpreguntas es guiar al estudiante a encontrar la respuesta correcta. Las preguntas son las siguientes:

1. ¿Cuál es su composición y su estructura?

2. ¿Cuáles son sus funciones?

3. ¿Está su función relacionada y/o determinada por su estructura? ¿Por qué crees que es así?

4. ¿Cómo afectan las malformaciones del citoesqueleto a la salud humana?

El contenido del CIMA está resumido en el mapa de contenidos (figura 1). En azul oscuro se muestran los tres bloques conceptuales en los que está dividido el CIMA. En azul claro se representan los contenidos que vertebran cada bloque. Las preguntas están coloreadas en rosa, rosa oscuro corresponde a la pregunta principal y rosa claro a las subpreguntas que guiarán el trabajo. El amarillo claro se refiere a los conceptos actitudinales, que en realidad engloban toda la actividad. Las dobles flechas indican interacciones entre ambos contenidos conceptuales, que es necesario remarcar para que los estudiantes entiendan que la función última del citoesqueleto es consecuencia de su composición y estructura.

Ciclos de Mejora en el Aula (2020). Experiencias de Innovación Docente de la US Esta obra se distribuye con la licencia Creative Commons 


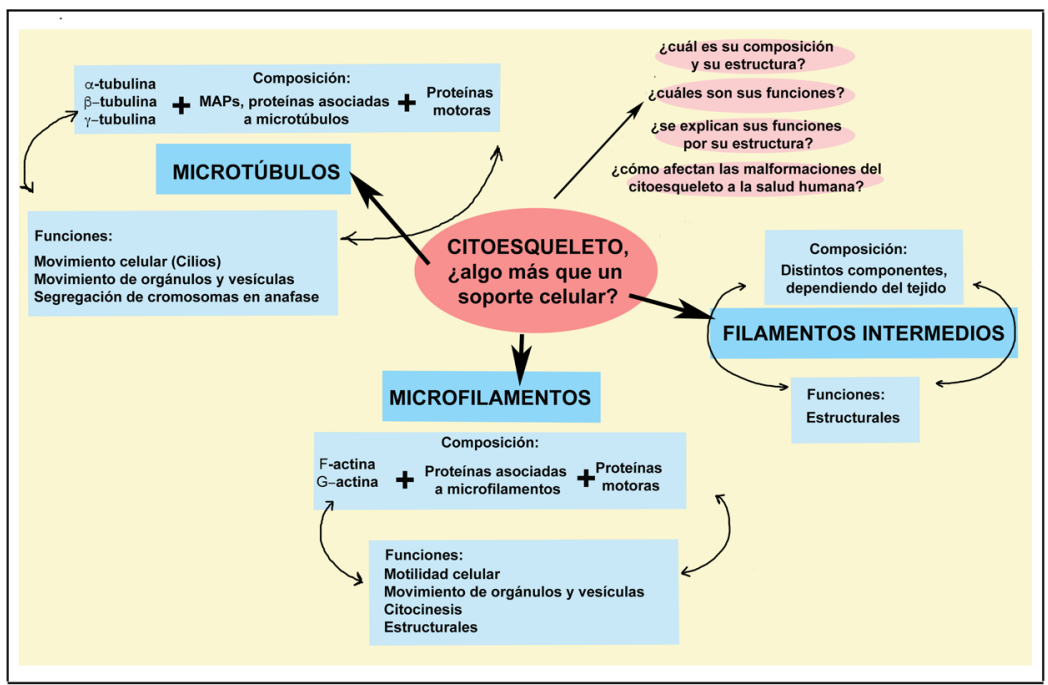

Figura 1. Mapa de contenidos

\section{Modelo metodológico posible}

A partir de una pregunta inicial (I) global los alumnos comienzan a trabajar en grupo a través de la búsqueda de información para realizar el proyecto $(\mathrm{Pr})$ que se les ha requerido. Este proyecto lo realizan guiados por las subpreguntas que aparecen en el mapa de contenidos y siempre con el propósito de que adquieran un conocimiento conceptual específico, marcado en azul en el mapa de contenidos. Una vez que los alumnos han elaborado su proyecto, se realiza la primera actividad de contraste (AC) que consiste en la exposición del mismo por parte de los estudiantes y debate posterior entre los estudiantes que exponen el trabajo y la profesora/resto de estudiantes. Tras esta actividad hay una pequeña sintesis (S) por parte de la profesora, donde se hace énfasis en las cuestiones que pudieron quedar menos clarificadas en la exposición de los alumnos. Hay varias actividades de contraste dentro de cada bloque temático que se trabaja (tal y como se muestra en la secuencia de actividades, tabla 1) y la Ciclos de Mejora en el Aula (2020). Experiencias de Innovación Docente de la US 
dinámica es la misma para todos los bloques. El modelo metodológico posible para este CIMA se representa en la figura 2.

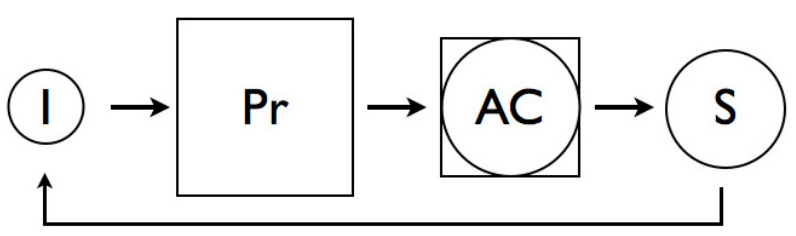
I Introducción al tema
AC Actividad de contraste
Pr Proyecto
$\mathrm{S}$ Conclusiones y síntesis
Actividad profesor
$\bigcirc$ Actividad conjunta
Actividad alumnos

Figura 2. Modelo metodológico posible para el ciclo de mejora en el aula

\section{Secuencias de actividades}

En esta sección se muestran las actividades programadas para cada uno de los tres bloques incluidos en el CIMA (tabla1). El CIMA comienza con el cuestionario inicial para evaluar los conocimientos y esquemas mentales de los alumnos previos a las actividades programadas. En estas sesiones se intercalan actividades realizadas por los alumnos individualmente o en grupo, con actividades conjuntas profesora-alumnos. Finaliza con una sesión de conclusiones-síntesis y con la realización del cuestionario final, idéntico al inicial.

Tabla 1. Secuencia de actividades programadas para el CIMA

\begin{tabular}{|l|l|l|}
\hline \multicolumn{1}{|c|}{ Actividad } & \multicolumn{1}{|c|}{ Descripción } & \multicolumn{1}{c|}{ Finalidad } \\
\hline Cuestionario inicial & Los alumnos realizan & Evaluar los \\
& un cuestionario de 5 & conocimientos previos \\
& preguntas relacionados & de los alumnos, antes \\
& con los temas que se & de comenzar el bloque \\
& van a trabajar en el & de temas sobre los que \\
& CIMA. & trabajaremos. \\
\hline
\end{tabular}

Ciclos de Mejora en el Aula (2020). Experiencias de Innovación Docente de la US Esta obra se distribuye con la licencia Creative Commons 


\begin{tabular}{|c|c|c|}
\hline Introducción & $\begin{array}{l}\text { Pequeña introducción } \\
\text { al tema y explicación } \\
\text { de la primera actividad. }\end{array}$ & $\begin{array}{l}\text { Informar a los alumnos } \\
\text { sobre el formato y } \\
\text { contenido del proyecto } \\
\text { que realizarán en } \\
\text { grupos. }\end{array}$ \\
\hline Proyecto 1 & $\begin{array}{l}\text { Los alumnos, en } \\
\text { grupos, preparan una } \\
\text { presentación sobre el } \\
\text { tema para exponer en } \\
\text { clase. Siguen preguntas } \\
\text { guía, artículos, } \\
\text { referencias facilitadas } \\
\text { por el profesor. }\end{array}$ & $\begin{array}{l}\text { Con esta actividad los } \\
\text { alumnos adquirirán un } \\
\text { conjunto de contenidos } \\
\text { teóricos relacionados } \\
\text { con los temas elegidos. }\end{array}$ \\
\hline $\begin{array}{l}\text { 1a actividad de } \\
\text { contraste }\end{array}$ & $\begin{array}{l}\text { Los alumnos presentan } \\
\text { el trabajo en clase y } \\
\text { después se debate con } \\
\text { preguntas, comentarios, } \\
\text { aclaraciones, etc, } \\
\text { realizadas tanto por el } \\
\text { profesor como por los } \\
\text { alumnos. }\end{array}$ & $\begin{array}{l}\text { Estimular la curiosidad } \\
\text { de los alumnos, } \\
\text { incitarles a participar. } \\
\text { Contrastar el } \\
\text { conocimiento previo } \\
\text { a la actividad con el } \\
\text { adquirido durante } \\
\text { la preparación del } \\
\text { proyecto } 1 .\end{array}$ \\
\hline $\begin{array}{l}\text { 2a actividad de } \\
\text { contraste }\end{array}$ & $\begin{array}{l}\text { Los alumnos } \\
\text { elaboran un cuadro } \\
\text { de calificación sobre } \\
\text { distintos aspectos de } \\
\text { la presentación de los } \\
\text { grupos. } \\
\text { El profesor debate con } \\
\text { los alumnos sobre } \\
\text { estos aspectos. }\end{array}$ & $\begin{array}{l}\text { Estimular la capacidad } \\
\text { crítica de los alumnos } \\
\text { ya que tienen que } \\
\text { evaluar el trabajo } \\
\text { hecho por otros. }\end{array}$ \\
\hline Proyecto 2 & $\begin{array}{l}\text { Los alumnos de forma } \\
\text { individual eligen una } \\
\text { patología humana } \\
\text { donde se centran en } \\
\text { buscar la conexión con } \\
\text { una malformación en } \\
\text { algún componente del } \\
\text { citoesqueleto, basados } \\
\text { en un modelo que el } \\
\text { profesor previamente } \\
\text { les facilita. }\end{array}$ & $\begin{array}{l}\text { Con esta actividad los } \\
\text { alumnos identifican } \\
\text { conexiones entre un } \\
\text { contenido puramente } \\
\text { teórico aprendido en } \\
\text { clase y situaciones } \\
\text { de la vida real, como } \\
\text { son las enfermedades } \\
\text { humanas. }\end{array}$ \\
\hline
\end{tabular}

Ciclos de Mejora en el Aula (2020). Experiencias de Innovación Docente de la US Esta obra se distribuye con la licencia Creative Commons Reconocimiento-NoComercial-SinObraDerivada Internacional (CC BY-NC-ND 4.0.) 


\begin{tabular}{|c|c|c|}
\hline $\begin{array}{l}\text { 3a actividad de } \\
\text { contraste }\end{array}$ & $\begin{array}{l}\text { Los alumnos presentan } \\
\text { la actividad online } \\
\text { y el profesor realiza } \\
\text { preguntas relacionadas } \\
\text { con las patologías } \\
\text { presentadas. }\end{array}$ & $\begin{array}{l}\text { La presentación y las } \\
\text { preguntas tienen el } \\
\text { objetivo de despertar } \\
\text { debates motivadores } \\
\text { para los alumnos. }\end{array}$ \\
\hline Conclusiones & $\begin{array}{l}\text { El profesor elabora una } \\
\text { pequeña evaluación/ } \\
\text { informe de cada } \\
\text { proyecto } 1 \text { y lo envía a } \\
\text { los grupos. }\end{array}$ & $\begin{array}{l}\text { Identificación de } \\
\text { aspectos concretos } \\
\text { donde los estudiantes } \\
\text { deben mejorar, } \\
\text { por ejemplo en } \\
\text { la elaboración de } \\
\text { conceptos, elaboración } \\
\text { de presentaciones, o } \\
\text { corrigiendo conceptos } \\
\text { erróneos, etc. }\end{array}$ \\
\hline Sintesis & $\begin{array}{l}\text { El profesor proporciona } \\
\text { a los alumnos una } \\
\text { presentación con los } \\
\text { puntos clave de los } \\
\text { temas y recalcando } \\
\text { aquellos que pudieron } \\
\text { no quedar claros en las } \\
\text { presentaciones de los } \\
\text { alumnos. }\end{array}$ & $\begin{array}{l}\text { La sintesis acaba de } \\
\text { fortalecer los nuevos } \\
\text { conceptos adquiridos } \\
\text { y genera una jerarquía } \\
\text { entre aquellos } \\
\text { conceptos principales y } \\
\text { secundarios. }\end{array}$ \\
\hline Cuestionario final & $\begin{array}{l}\text { Los alumnos realizan } \\
\text { un cuestionario de } 5 \\
\text { preguntas idéntico al } \\
\text { inicial y relacionados } \\
\text { con los temas tratados. }\end{array}$ & $\begin{array}{l}\text { Evaluar los } \\
\text { conocimientos } \\
\text { posteriores de los } \\
\text { alumnos, después de } \\
\text { las sesiones de trabajo. }\end{array}$ \\
\hline
\end{tabular}

\section{Cuestionario inicial-final}

La evaluación en la enseñanza universitaria es uno de los pilares fundamentales que nos dan una medida de la calidad de nuestra enseñanza. La calidad irá necesariamente de la mano del nivel de aprendizaje de nuestros alumnos. La evaluación de ese aprendinzaje durante el CIMA la he realizado segun las pautas de Rivero y Porlán (2007). Antes de comenzar con las actividades programadas para el CIMA, los estudiantes han contestado a un cuestionario con el fin de poder evaluar su nivel de 
conocimiento inicial relacionado con el tema que se desarrolla en el CIMA, citoesqueleto celular. Los alumnos realizan el cuestionario online con un tiempo limitado para que no puedan consultar medios externos a sus propios conocimientos adquiridos con anterioridad a nuestra actividad en el aula. Tiene un carácter voluntario pero no anónimo, y se les explica que la contestación no influirá en la calificación final de la asignatura. Así sienten más libertad a la hora de contestar a las preguntas del cuestionario.

Al final del ciclo de mejora se les pide que vuelvan a rellenar el cuestionario, que es exactamente el mismo que al inicio. De esta manera, podemos valorar, al analizar ambos cuestionarios, cómo ha evolucionado cada alumno con estas sesiones.

El cuestionario consta de cinco preguntas, cada una intenta abarcar alguna cuestión básica de cada uno de los tres bloques teóricos que afectan al ciclo de mejora:

1. Enumera procesos celulares donde el citoesqueleto juegue un papel fundamental. Explica brevemente cuál es la función del citoesqueleto en cada uno de estos procesos.

2. ¿Qué componentes del citoesqueleto participan en crear, de una u otra manera, dinamismo a la célula? Describe lo más detalladamente posible la función concreta del citoesqueleto en estos procesos dinámicos.

3. ¿Crees que todos los centros organizadores de microtúbulos son centrosomas? Razona tu contestación.

4. ¿Crees que la mayor parte de las células eucariotas tienen cilios? Describe las funciones de los cilios.

5. Imagínate una célula epitelial que "nace" o se origina sin filamentos intermedios. ¿Cuáles serían las consecuencias?

Ciclos de Mejora en el Aula (2020). Experiencias de Innovación Docente de la US Esta obra se distribuye con la licencia Creative Commons 


\section{Aplicación del ciclo de mejora docente en el aula}

\section{¿Qué sucede en el aula durante la aplicación del CIMA?}

El trabajo individual y en grupo de los estudiantes ha sido satisfactorio durante estas sesiones especiales. La mayor parte de ellos participan en los cuestionarios y todos trabajan en los proyectos requeridos, con mayor o menor implicación. Para la realización del proyecto 1 cuentan con un par de semanas en las que trabajan en grupo sin mi supervisión directa, pero con la ayuda de las preguntas guía y de material que suministro a través de la enseñanza virtual. Algunos de los estudiantes manifiestan por correo electrónico dudas sobre qué contenidos incluir en el trabajo o cuestiones conceptuales. A pesar de que todo se desarrolla online, la comunicación con los estudiantes es fluida.

Durante la primera actividad de contraste, todos nos reunimos online durante $1 \mathrm{~h} 30 \mathrm{~min}$. En ellas, y de forma voluntaria, los estudiantes presentan sus trabajos de forma oral al resto de la clase. Al final de cada presentación, planteo cuestiones al estudiante que ha presentado con el objetivo de identificar y aclarar posibles errores conceptuales, o para profundizar en conceptos que no han quedado suficientemente trabajados en la presentación. Tras esto, doy paso a la participación de los estudiantes mediante cuestiones sobre lo expuesto. Aquí, noto que la experiencia online supone un freno para los estudiantes, que no participan activamente en la actividad.

Para mi sorpresa, observé más participación en la 3ạ actividad de contraste donde los estudiantes, futuros médicos, eligen, estudian y presentan una enfermedad o síndrome en humanos relacionado con el mal funcionamiento

Ciclos de Mejora en el Aula (2020). Experiencias de Innovación Docente de la US Esta obra se distribuye con la licencia Creative Commons 
del citoesqueleto celular. En una de estas sesiones, acabamos con una discusión muy animada, por chat y/o micrófonos abiertos, sobre la base genética del envejecimiento, tema que en principio se salía del ámbito de la patología en la que se centraba la sesión. Sin embargo, como digo se encendió la curiosidad de varios de los estudiantes. Mi interpretación es que para despertar el interés de los alumnos tenemos que hacer las preguntas adecuadas, preguntas motivadoras, preguntas que puedan ligar la materia con sus intereses vitales, en este caso enfermedades humanas ya que son futuros médicos. Esta es una vía muy poderosa de captar su atención, y por lo tanto muy efectiva para el proceso de aprendizaje (Bain, 2004).

Como aspecto menos positivo del modelo metodológico aplicado en este CIMA es que presenta una limitación importante cuando se trabaja con grupos de clase muy numerosos. En mi caso, el grupo lo componen alrededor de 60 alumnos. Para la realización de la actividad, los alumnos se agruparon en 3-4 personas, por lo que había unos 15 grupos. Esto ha limitado la participación de la gran mayoría de los estudiantes en la presentación oral. Además, intento comentar individualmente a cada grupo aspectos concretos que deben mejorar. Para que esta actividad funcione se requieren menor número de grupos o disponer de sesiones más largas.

\section{Evaluación del aprendizaje de los estudiantes tras la aplicación del CIMA}

Como ya he comentado, la mayoría de los alumnos participaron en los cuestionarios, algunos solo realizaron el inicial, otros el final, 23 alumnos realizaron ambos. Me he centrado en los 23 que participaron al inicio y al final para analizar sus respuestas a cada una de las 5 preguntas propuestas.

Ciclos de Mejora en el Aula (2020). Experiencias de Innovación Docente de la US Esta obra se distribuye con la licencia Creative Commons 
Para cada una de las preguntas, he establecido tres niveles de respuestas o escalones en base a las respuestas dadas en su conjunto por los alumnos (niveles establecidos a posteriori). Para establecer estos niveles se tiene en cuenta el grado de complejidad de la respuesta y el grado de relación de unos conceptos con otros. En la tabla 2, se explican los niveles para la pregunta 1 en particular. En la figura 3, aparecen reflejados el porcentaje de estudiantes en cada nivel, antes y después del cima, y para cada una de las cinco preguntas.

Tabla 2. Niveles de respuesta para la pregunta 1 del cuestionario

\begin{tabular}{|l|l|}
\hline NIVEL & Respuesta a la PREGUNTA1 \\
\hline 1 (escalón inferior) & $\begin{array}{l}\text { No contesta, es errónea, conocimiento básico sin } \\
\text { conexión entre elementos del citoesqueleto. Sabe } \\
\text { identificar alguna función del citoesqueleto. }\end{array}$ \\
\hline 2 (escalón medio) & $\begin{array}{l}\text { Sabe identificar una variedad de funciones } \\
\text { y además las relaciona con elementos del } \\
\text { citoesqueleto particulares. }\end{array}$ \\
\hline 3 (escalón superior) & $\begin{array}{l}\text { Identifica funciones del citoesqueleto donde } \\
\text { intervienen varios elementos del mismo. El } \\
\text { alumno tiene un conocimiento en forma de red, } \\
\text { donde los nódulos están conectados entre ellos y } \\
\text { con elementos secundarios de conocimiento. }\end{array}$ \\
\hline
\end{tabular}

Para todas la preguntas, se observa que tras las sesiones los alumnos en su conjunto pudieron responder con más complejidad y profundidad. Sin embargo, no sabemos qué hubiera sucedido si la misma evaluación se hubiera hecho tras la aplicación del modelo habitual de enseñanza (nos falta el control interno del experimento). En una asignatura como Biología, sin grandes contenidos abstractos, hemos observado que habitualmente los estudiantes aprenden tras recibir clases magistrales típicas. ¿Hemos conseguido con este CIMA un aprendizaje duradero y real? Sería interesante volver a hacer el cuestionario pasados unos meses tras la implementación del CIMA.

Ciclos de Mejora en el Aula (2020). Experiencias de Innovación Docente de la US Esta obra se distribuye con la licencia Creative Commons 
El cuadro de evolución por estudiante (Tabla 3) muestra la evolución de cada estudiante para cada pregunta. Si el estudiante ha contestado mejor el cuestionario final que el inicial, tendrá un tono más intenso de color. Si por el contrario, la evolución no es favorable, el color será más tenue. Si el cuadro no tiene color, no se ha podido observar evolución en el estudiante. Los resultados muestran que en algunas de las preguntas los alumnos no han aprendido tanto como se esperaba. Esto puede ser debido a dos factores: que el nivel de los estudiantes era inicialmente muy alto o que el método de enseñanza no ha funcionado muy bien. Sin embargo, en general ha habido un cambio muy positivo en la mayoría de los alumnos.

El cuadro no muestra, sin embargo, el grado de evolución, solo si es positiva, negativa o neutra. Tampoco refleja el nivel relativo de conocimiento de cada alumno con respecto al resto de compañeros, solo si el método ha servido para que aprenda algo o no, en neto.

En resumen, solo he detectado un alumno donde no se ha observado prácticamente aprendizaje (estudiante 17). El resto ha respondido favorablemente al método y han adquirido nuevos conocimientos, que esperemos que les sirvan para aprender.

Ciclos de Mejora en el Aula (2020). Experiencias de Innovación Docente de la US Esta obra se distribuye con la licencia Creative Commons 


\section{SITUACIÓN PREVIA}

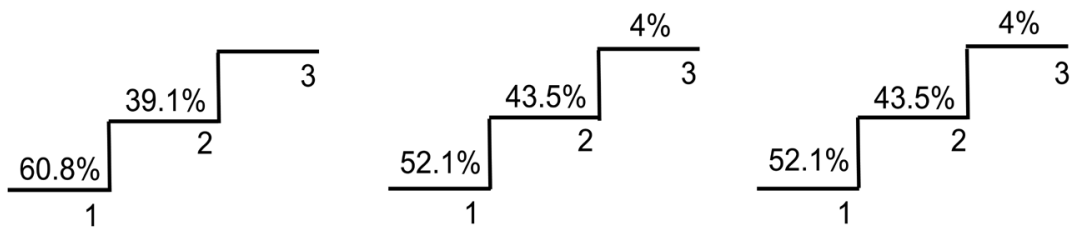

\section{SITUACIÓN POSTERIOR}

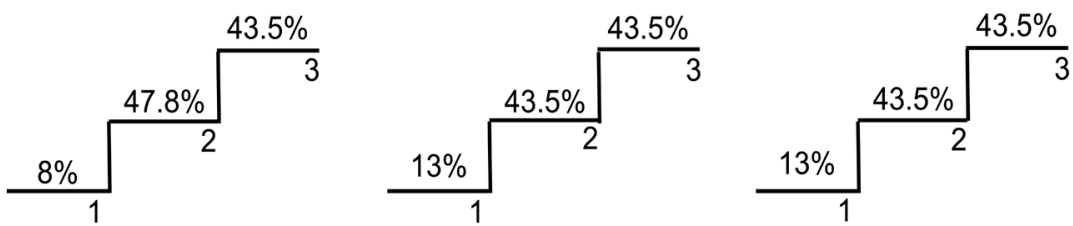

PREGUNTA 4

PREGUNTA 5

SITUACIÓN PREVIA

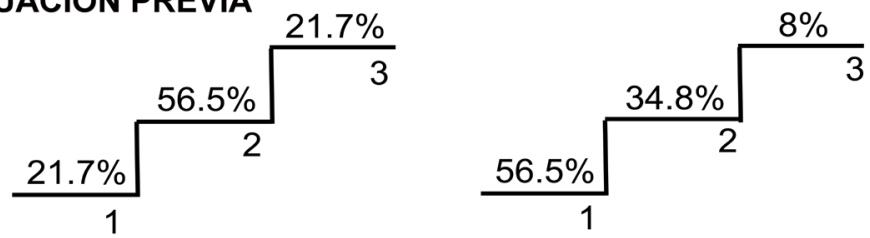

\section{SITUACIÓN POSTERIOR}

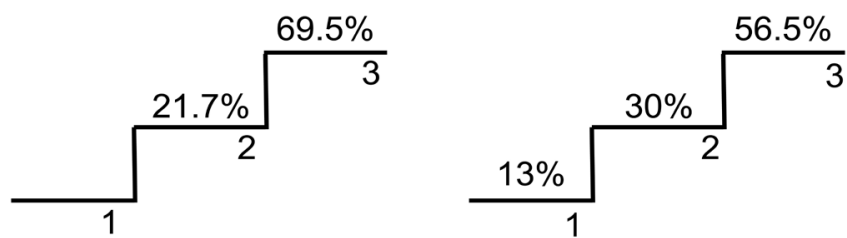

Figura 3. Porcentaje de estudiantes en cada nivel, para cada pregunta, antes (situación previa) y después (situación posterior) del CIMA.

Ciclos de Mejora en el Aula (2020). Experiencias de Innovación Docente de la US Esta obra se distribuye con la licencia Creative Commons 
Tabla 3. Cuadro de evolución de cada estudiante en las 5 preguntas del cuestionario.

\begin{tabular}{|c|c|c|c|c|c|}
\hline Estudiante & Pregunta 1 & Pregunta 2 & Pregunta 3 & Pregunta 4 & Pregunta 5 \\
\hline 1 & & & & & \\
\hline 2 & & & & & \\
\hline 3 & & & & & \\
\hline 4 & & & & & \\
\hline 5 & & & & & \\
\hline 6 & & & & & \\
\hline 7 & & & & & \\
\hline 8 & & & & & \\
\hline 9 & & & & & \\
\hline 10 & & & & & \\
\hline 11 & & & & & \\
\hline 12 & & & & & \\
\hline 13 & & & & & \\
\hline 14 & & & & & \\
\hline 15 & & & & & \\
\hline 16 & & & & & \\
\hline 17 & & & & & \\
\hline 18 & & & & & \\
\hline 19 & & & & & \\
\hline 20 & & & & & \\
\hline 21 & & & & & \\
\hline 22 & & & & & \\
\hline 23 & & & & & \\
\hline
\end{tabular}

Ciclos de Mejora en el Aula (2020). Experiencias de Innovación Docente de la US Esta obra se distribuye con la licencia Creative Commons Reconocimiento-NoComercial-SinObraDerivada Internacional (CC BY-NC-ND 4.0.) 


\section{Evaluación del CIMA puesto en práctica}

\section{¿Qué aspectos cambiaría, mantendría o mejoraría en futuros CIMAs?}

Una de las cuestiones que me ha llamado la atención en este ciclo de mejora es que, además de cambiar nuestros hábitos metodológicos y acercarnos al estudiante, también es sumamente importante priorizar los contenidos en nuestras clases. Este es un tema especialmente sensible en el campo de la biología, donde nuevos descubrimientos van aportando cada día nuevos y más profundos conocimientos del funcionamiento de la célula. Ya que el tiempo de cada curso es limitado y sin embargo la materia crece sin cesar, es importante separar lo fundamental de lo accesorio y priorizar los contenidos. Este CIMA me ha ayudado a priorizar contenidos, gracias a una herramienta que me ha parecido fundamental como es el mapa de contenidos. La construcción de mapas de contenidos es una actividad que me gustaría incorporar en mi actividad docente de forma habitual.

Las actividades de contraste también son fundamentales en el proceso de aprendizaje. Sin embargo, en cursos con un elevado número de alumnos tienen su limitación pero sin duda son actividades muy interesantes a incluir al menos en algún bloque de la asignatura. Tal vez, se pueden organizar actividades de contraste más dinámicas, intercaladas con las clases con modelos más tradicionales, en forma de preguntas lanzadas directamente a los alumnos en la clase, que capten su atención y activen su motivación para aprender.

Ciclos de Mejora en el Aula (2020). Experiencias de Innovación Docente de la US Esta obra se distribuye con la licencia Creative Commons 


\section{Principios didácticos que han guiado mi experiencia y que permanecerán en el futuro}

Resumo aquí algunos de los principios didácticos que me han ayudado a implementar este CIMA y que espero que permanezcan en mis futuras experiencias docentes:

- Teoría sobre el conocimiento: dada mi experiencia investigadora en el campo de la Biología, entiendo el conocimiento científico como un conocimiento cambiante, que evoluciona con el tiempo. Nuevos descubrimientos de la comunidad científica validan o descartan hipótesis de trabajo previas. Este carácter relativo y provisional del conocimiento en biología lo intento trasmitir a mis estudiantes continuamente.

- El profesor no es la persona donde reside la verdad absoluta: en relación a lo anterior, intento trasmitir a los estudiantes que el profesor, aunque debe conocer muy bien la materia que enseña, en ocasiones no puede abarcar todo este inmenso y cambiante mundo de la biología. Es bueno que los alumnos entiendan que ellos mismos pueden aportar a la clase conocimientos, son parte activa del proceso de aprendizaje que se produce en el aula también en este aspecto.

- En cuanto a los contenidos: como he mencionado anteriormente, es fundamental priorizar contenidos y jerarquizarlos. Lo importante no se debe confundir con lo secundario. También es necesario fomentar la adquisición no solo de contenidos conceptuales sino también actitudinales, como el espíritu crítico, tan necesario en nuestra vida cotidiana, y en particular en las ciencias experimentales.

- Metodología: implementar una metodología acorde a nuestro momento, a la cotidianidad de los estudiantes. Sacar partido de los avances tecnológicos para adaptar aquello que pueda mejorar nuestra enseñanza.

- Participación del estudiante de forma activa en el proceso de aprendizaje: este es uno de los principios didácticos más poderosos, al menos en mi experiencia en la aplicación de este CIMA.

Ciclos de Mejora en el Aula (2020). Experiencias de Innovación Docente de la US Esta obra se distribuye con la licencia Creative Commons 
Palabras claves: Biología, Grado en Medicina, Docencia Universitaria, Experimentación docente universitaria, Citoesqueleto celular, Mapa de Contenidos, Principios Didácticos.

Key words: Biology, Degree in Medicine, University Teaching, University Teaching Experimentation, Cellular Cytoskeleton, Content Map, Didactic Principles.

\section{Referencias Bibliográficas}

Bain, K. (2004). Lo que hacen los mejores profesores universitarios. Valencia: Universitat de Valencia.

Finkel, D. (2008). Dar clase con la boca cerrada. Valencia: Universitat de Valencia.

García-Díaz, E., Porlán, R., y Navarro, E. (2017). Los fines y los contenidos de enseñanza. En Porlán (Coord.), Enseñanza Universitaria. Cómo mejorarla (pp. 55-72). Madrid: Morata.

Rivero, A. y Porlán, R. (2017). La evaluación en la enșeñanza universitaria. En Porlán (Coord.), Enseñanza Universitaria. Cómo mejorarla (pp. 73-91). Madrid: Morata.

Ciclos de Mejora en el Aula (2020). Experiencias de Innovación Docente de la US Esta obra se distribuye con la licencia Creative Commons 